\title{
A detached petal disc assay and virus-induced gene silencing facilitate the study of Botrytis cinerea resistance in rose flowers
}

\author{
Xiaogian Cao ${ }^{1}$, Huijun Yan², Xintong Liu', Dandan $\mathrm{Li}^{1}$, Mengjie Sui ${ }^{2}$, Jie $\mathrm{Wu}^{1}$, Hongqiang $\mathrm{Yu}^{1}$ and Zhao Zhang (i)
}

\begin{abstract}
Fresh-cut roses (Rosa hybrida) are one of the most important ornamental crops worldwide, with annual trade in the billions of dollars. Gray mold disease caused by the pathogen Botrytis cinerea is the most serious fungal threat to cut roses, causing extensive postharvest losses. In this study, we optimized a detached petal disc assay (DPDA) for artificial B. cinerea inoculation and quantification of disease symptoms in rose petals. Furthermore, as the identification of rose genes involved in B. cinerea resistance could provide useful genetic and genomic resources, we devised a virusinduced gene silencing (VIGS) procedure for the functional analysis of $B$. cinerea resistance genes in rose petals. We used RhPR10.1 as a reporter of silencing efficiency and found that the rose cultivar 'Samantha' showed the greatest decrease in RhPR10.1 expression among the cultivars tested. To determine whether jasmonic acid and ethylene are required for $B$. cinerea resistance in rose petals, we used VIGS to silence the expression of RhLOXS and RhEIN3 (encoding a jasmonic acid biosynthesis pathway protein and an ethylene regulatory protein, respectively) and found that petal susceptibility to $B$. cinerea was affected. Finally, a VIGS screen of $B$. cinerea-induced rose transcription factors demonstrated the potential benefits of this method for the high-throughput identification of gene function in B. cinerea resistance. Collectively, our data show that the combination of the DPDA and VIGS is a reliable and highthroughput method for studying $B$. cinerea resistance in rose.
\end{abstract}

\section{Introduction}

Fresh cut roses are among the most popular ornamental plants, generating billions of dollars in trade annually and accounting for more than one-third of the total cut flower industry worldwide ${ }^{1}$. The supply of cut roses is heavily reliant on the logistics of long-distance transportation, as cut roses are produced mainly in developing countries with low labor costs and suitable climates, such as Kenya, Colombia, Ecuador, and China, whereas the consumer market is mainly in the developed countries of North America, Europe, and Japan. Globally, the average

\footnotetext{
Correspondence: Zhao Zhang (zhangzhao@cau.edu.cn)

${ }^{1}$ Beijing Key Laboratory of Development and Quality Control of Ornamental

Crops, Department of Ornamental Horticulture, College of Horticulture, China Agricultural University, Beijing 100193, China

${ }^{2}$ Flower Research Institute of Yunnan Academy of Agricultural Sciences, Kunming 650205 Yunnan, China

These authors contributed equally: Xiaoqian Cao, Huijun Yan, Xintong Liu
}

transport distance for each rose, from farm to customer, is more than $1500 \mathrm{~km}^{2}$. During this long-distance transportation, cut roses are challenged by various biotic and abiotic stresses, among which gray mold disease caused by Botrytis cinerea causes the most severe postharvest losses.

B. cinerea is among the world's most notorious plant pathogens, causing gray mold disease in over 200 dicotyledonous and monocotyledonous species ${ }^{3}$. Germinated B. cinerea conidia produce secondary metabolites and phytotoxic proteins that induce host cell death during the penetration of the host epidermis ${ }^{4}$. In rose, B. cinerea infection leads to necrotic lesions on petals, and symptoms develop rapidly during postharvest transport, during which flowers are packed in boxes with high relative humidity ${ }^{5}$.

Despite the economic importance of this pathogen in roses, research on the rose- $B$. cinerea interaction has

\section{(c) The Author(s) 2019}

(c) Open Access This article is licensed under a Creative Commons Attribution 4.0 International License, which permits use, sharing, adaptation, distribution and reproduction cc. in any medium or format, as long as you give appropriate credit to the original author(s) and the source, provide a link to the Creative Commons license, and indicate if changes were made. The images or other third party material in this article are included in the article's Creative Commons license, unless indicated otherwise in a credit line to the material. If material is not included in the article's Creative Commons license and your intended use is not permitted by statutory regulation or exceeds the permitted use, you will need to obtain permission directly from the copyright holder. To view a copy of this license, visit http://creativecommons.org/licenses/by/4.0/. 
been limited compared to research on the pathogen's behavior in other plants, such as the model plant Arabidopsis thaliana (Arabidopsis) and the Solanaceous species Nicotiana benthamiana and tomato (Solanum lycopersicum), which are also susceptible to $B$. cinerea. However, research on $B$. cinerea infection in Arabidopsis and $N$. benthamiana has focused on the infection of leaves and, in tomato, that of fruits. In ornamental crops such as gerbera $^{6}$ and rose ${ }^{4,7}$ by contrast, $B$. cinerea mainly infects flower petals, damaging the most economically important organ of these plants, while the leaves, fruits, stems, and sepals are rarely infected or of little importance. Petal cells begin to senesce immediately after harvest ${ }^{8}$, which facilitates infection by necrotrophic fungi such as $B$. cinerea. In addition, petal epidermal cells are a unique plant tissue with specialized conical structures. The valleys between conical cells may facilitate the survival of airborne B. cinerea conidia.

Artificial inoculation is a critical technique in disease phenotyping and thus in studies of the interaction between $B$. cinerea and rose, both for fundamental research and for breeding purposes. Previously, rose was inoculated with $B$. cinerea via the inoculation of whole flowers with fungal conidia; disease severity was scored according to a "disease index" based on a scale of $0-5$ (or more), ranging from no infection to the fungus covering the whole flower ${ }^{9,10}$. However, this conventional method cannot be used to accurately quantify disease resistance. We therefore aimed to develop an improved method for artificial inoculation and disease quantification in rose petals. To this end, we designed and optimized a detached petal disc assay (DPDA) for artificial inoculation and accurate symptom quantification of $B$. cinerea infection in rose.

Furthermore, as the functional characterization of rose genes thought to be involved in B. cinerea resistance is limited by the low efficiency and long timeframe of genetic transformation (2 years from transformation to flower production), we used an alternative molecular approach involving virus-induced gene silencing (VIGS). This method, in which target genes are knocked down based on double-stranded RNA-triggered RNA degradation, has been widely exploited for gene functional analysis ${ }^{11}$. In VIGS, when a recombinant virus carrying the sequence of a host genelspreads throughout the plant, the host target gene transcripts are degraded together with the viral transcripts, and the gene of interest in the host plant is therefore silenced. Previously, a VIGS approach using recombinant tobacco rattle virus (TRV) was established for the silencing of genes in rose flowers ${ }^{12}$. Once young plantlets or sprouts are vacuum infiltrated with Agrobacterium tumefaciens (Agrobacterium) carrying recombinant TRV-derived vectors, the infected plantlets/ sprouts are grown in soil or grafted to a root stock until flowering (which takes approximately 5-15 weeks), at which point it is possible to evaluate their phenotypes, such as flower color, scent, and floral development ${ }^{12}$. Although it has not been reported previously, it is likely that this VIGS protocol could be used to study rose resistance to pathogens. However, the full exploitation of VIGS assays for investigating $B$. cinerea resistance in rose petals could be hampered by the time ( $5-15$ weeks) and labor costs of VIGS. In addition, a large volume of the Agrobacterium suspension is needed for the immersion and vacuum infiltration of plantlets, and a large area in a greenhouse or climate-controlled chamber is required to support the growth of rose plants from agroinfiltration until flowering. These limitations have prevented the rapid and high-throughput screening of $B$. cinerea resistance genes in rose flowers.

To address these challenges, we developed a VIGS method for silencing candidate genes in detached rose petal discs, which allows the rapid identification of their function. We demonstrate that this VIGS method, combined with the DPDA, can be used for high-throughput screening of candidate $B$. cinerea resistance genes.

\section{Results \\ Inoculation of detached rose petal discs with $B$. cinerea}

To establish the DPDA, we obtained detached petals from the outermost whorl of rose (Rosa hybrida) flowers at stage 2 of flower opening ${ }^{13}$ and punched out $15-\mathrm{mm}$ discs from the center of the petals. We placed the petal discs on a wet filter paper in a 9-cm petri dish and inoculated the abaxial or adaxial surface of the discs with $2 \mu \mathrm{L}$ of $B$. cinerea conidia at $1 \times 10^{4} / \mathrm{mL}$. However, we observed only tiny disease spots up to $72 \mathrm{~h}$ postinoculation (hpi). This may have been due to the difficulty of controlling the wetness of the filter paper under this method, which resulted in insufficient humidity for $B$. cinerea infection (Supplemental Fig. S1a).

To overcome this problem, we placed petal discs on $0.4 \%(\mathrm{w} / \mathrm{v})$ agar and inoculated the discs using the same method. Through this approach, we observed clear, extended, and consistent lesions on all inoculated discs on either the adaxial or abaxial side at 48 hpi (Fig. 1a; Supplemental Fig. S1b). We subsequently inoculated the adaxial side of discs on $0.4 \%(\mathrm{w} / \mathrm{v})$ agar.

To determine the optimal concentration of fungal spores for inoculation as well as the optimal time point for phenotyping, we inoculated petal discs with $1 \times 10^{4} / \mathrm{mL}$, $1 \times 10^{5} / \mathrm{mL}$, and $1 \times 10^{6} / \mathrm{mL}$ conidia and measured disease lesions at 12, 24, 36, 48, 60, and $72 \mathrm{hpi}$. At 60 and $72 \mathrm{hpi}$, we observed statistically significant differences in the disease symptoms (lesion diameter) of discs inoculated with the three different conidia concentrations (Fig. 1b). In addition, we observed ion leakage resulting from petal cell death, consistent with the diameters of the lesions 

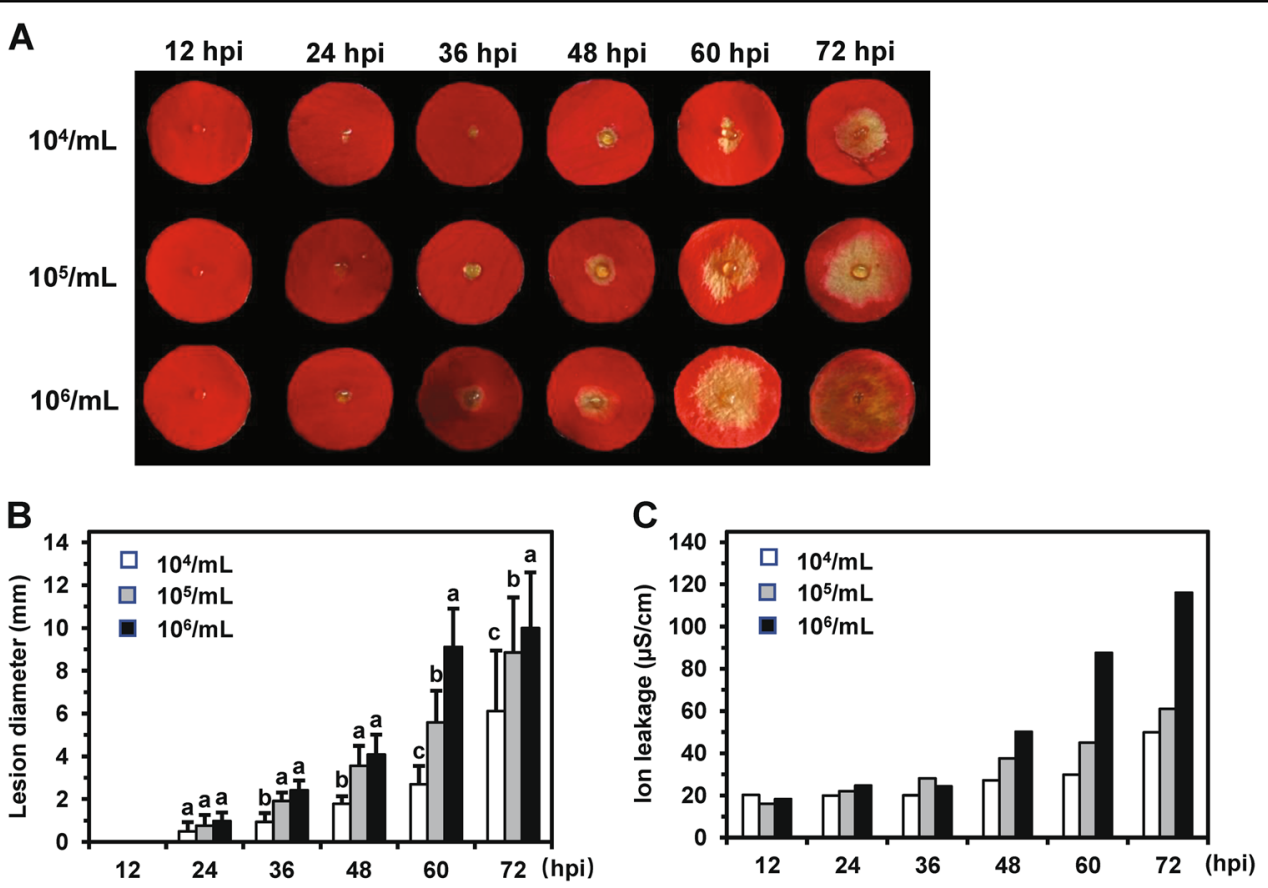

Fig. 1 Optimization of Botrytis cinerea inoculation on rose petal discs. a Petal discs were inoculated with different concentrations of $B$. cinerea conidia. Disease lesions were observed at $12,24,36,48,60$, and 72 hpi. b Statistical analysis of disease lesions following inoculation with different concentrations of $B$. cinerea conidia. The graph shows the average lesion size from three biological replicates $(n=48)$ with the standard deviation. Significant differences are indicated by lowercase letters according to Duncan's multiple range test $(P<0.05)$. c Quantification of cell death triggered by $B$. cinerea infection of rose petal discs. The graph shows ion leakage values measured at $12,24,36,48,60$, and $72 \mathrm{hpi}$. The ion leakage changes resulting from petal cell death were consistent with the disease lesion diameter. This experiment was repeated two times with similar results.

(Fig. 1c). This further suggested that although punching out a disc causes mechanical damage and, hence, some ion leakage, the cell death induced by $B$. cinerea further affects the ion leakage of petals. For the DPDA, we ultimately decided to use $2 \mu \mathrm{L}$ of conidia at $1 \times 10^{5} / \mathrm{mL}$ delivered to the adaxial side of the petal discs and to measure and statistically analyze disease lesions at $60 \mathrm{hpi}$.

\section{Characterization of the disease susceptibility of 22 rose genotypes}

Using the DPDA, we investigated disease susceptibility in a collection of rose cultivars. To this end, we used three cultivars bred by our lab as controls: 'Beijinghong', which is relatively resistant to $B$. cinerea, and 'Meirenxiang' and 'Xiang Fei', which are susceptible cultivars based on our observations in the field. We showed that the lesions of 'Beijinghong' exhibited an average diameter of less than $5 \mathrm{~mm}$ at $60 \mathrm{hpi}$, indicative of significantly more resistance than in the two susceptible cultivars (both of which exhibited lesions with average diameters over $12 \mathrm{~mm}$; Fig. 2). The results of the DPDA for 'Beijinghong', 'Xiangfei', and 'Meirenxiang' were consistent with their susceptibility observed in the field, suggesting that the lesion diameter observed by the DPDA is a good representation of flower fungal resistance.
We further tested a collection of 19 rose cultivars and found substantial differences in their susceptibility to $B$. cinerea. The cultivar 'Lvyu' was the most resistant, with an average lesion size at $60 \mathrm{hpi}$ of less than $3 \mathrm{~mm}$; some cultivars, such as 'Black Magic', 'Samantha', 'Red Naomi', 'Avalanche', and 'Carola', were moderately susceptible, with an average lesion size of $5-8 \mathrm{~mm}$; and others, including 'Perfume Delight', 'Hong Chun', and 'Vendela', were highly susceptible to $B$. cinerea, with lesion sizes of 12-15 mm (Fig. 2).

\section{TRV-based VIGS in detached rose petals}

It was previously demonstrated that TRV-mediated VIGS can be used in rose as a tool for the functional analysis of genes involved in flower development and the determination of petal color, and fragrance ${ }^{12}$. To assess the roles of candidate genes in B. cinerea resistance signaling, we aimed to optimize TRV VIGS by identifying rose cultivars that would be suitable for inoculation and efficient VIGS in petal discs. First, an appropriate cultivar should exhibit clear lesions after inoculation, as the edges of the lesions are not very clear in some cultivars, hampering their measurement (e.g., 'Marie-Victorin', Supplemental Fig. S2). Second, an ideal cultivar should be moderately resistant to $B$. cinerea, making it suitable for 
A

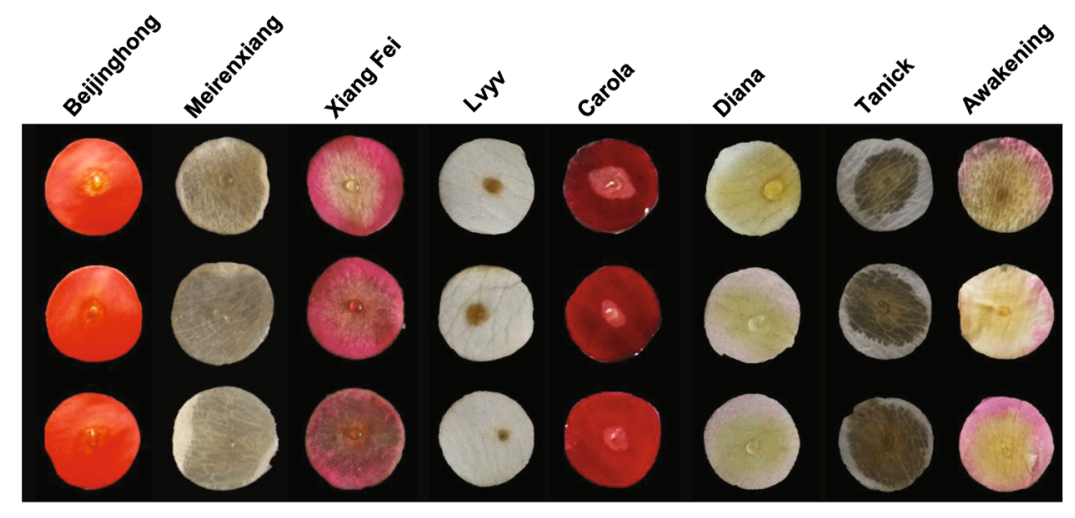

B

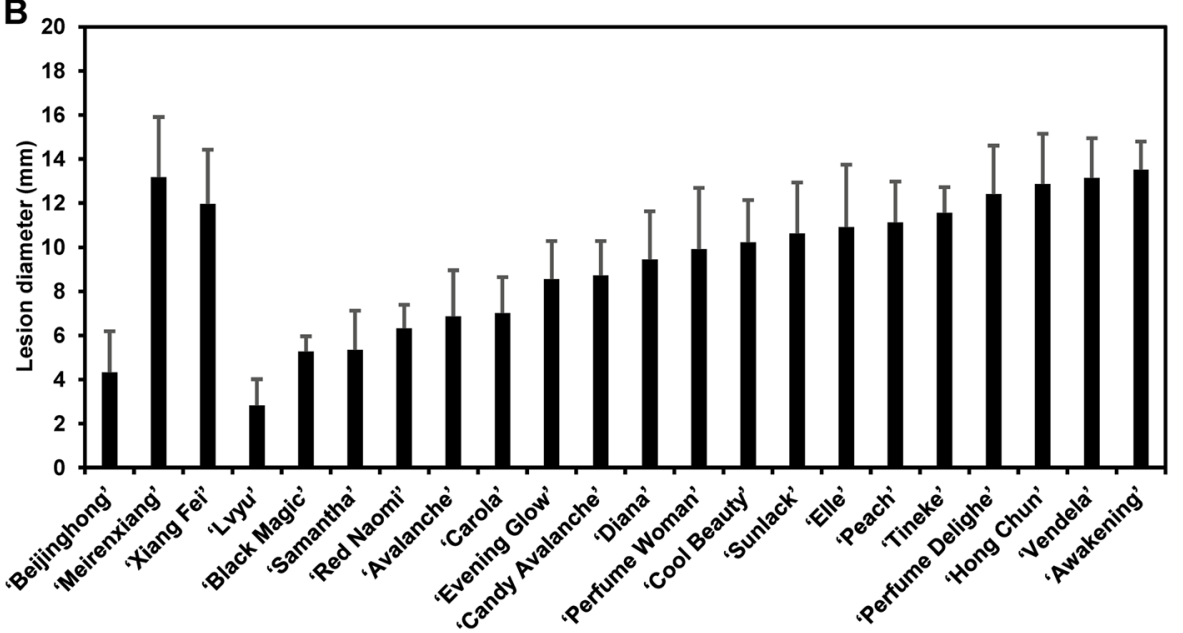

Fig. 2 Quantification of $B$. cinerea resistance in various rose cultivars determined using the detached petal disc assay. a Sample images of petal discs from each cultivar after infection with $B$. cinerea (photographed at $60 \mathrm{hpi}$ ). b Average lesion size measured at $60 \mathrm{hpi}$ from at least three biological replicates of each cultivar ( $N \geq 48$ for each cultivar) with the standard deviation.

the screening of both positive regulators (whose knockdown through gene silencing results in larger disease lesions) and negative regulators (whose knockdown results in smaller lesions) of defense signaling. Third, dark flowers are more suitable for the analysis than pale flowers because the fading of dark-colored petals caused by the silencing of anthocyanin-related genes can be used as a reporter of silencing efficiency (similar to the Phytoene Desaturase (PDS) gene, used as a reporter of silencing efficiency in plant leaves ${ }^{14}$.

We therefore selected four rose varieties with clear lesions, moderate resistance, and dark color-'Samantha', 'Carola', 'Black Magic', and 'Red Naomi'-as candidates for VIGS assays. Different cultivars may show different efficiencies of TRV-mediated gene silencing ${ }^{14,15}$. To test the efficiency of gene silencing in the four candidate cultivars, we used the RhDFR1 (Dihydroflavonol 4reductase 1) gene as a reporter. RhDFR1 is required for the accumulation of anthocyanin in rose flowers, and its silencing has been shown to lead to bleached flower color symptoms throughout rose flowers ${ }^{12}$. To determine whether the silencing of RhDFR1 induced similar bleaching in rose petal discs, we vacuum infiltrated a 1:1 mixture of Agrobacterium carrying $p T R V 1$ and $p T R V 2:$ : $R h D F R 1$ into rose petal discs. However, we did not observe increased color fading in the petals up to 10 days postinfiltration (Supplementary Fig. S3). It could be that the pigments have already accumulated in the petals of fully developed flowers and that the silencing of $R h D F R 1$ does not reduce the level of pigments already present in the petals. Thus, we concluded that, unlike the situation in complete flowers (Yan et al. ${ }^{12}$ ), RhDFR1 cannot be used as a silencing reporter gene for VIGS in petal discs.

Recently, a suppressor of petal senescence, RhPR10.1, was identified ${ }^{16}$. Knockdown of RhPR10.1 expression accelerates senescence in rose petals, resulting in a colorfading phenotype. To compare the silencing efficiency of this gene in the four candidate cultivars, we vacuum infiltrated Agrobacterium bacteria containing the recombinant TRV vectors into the petal discs of the four 

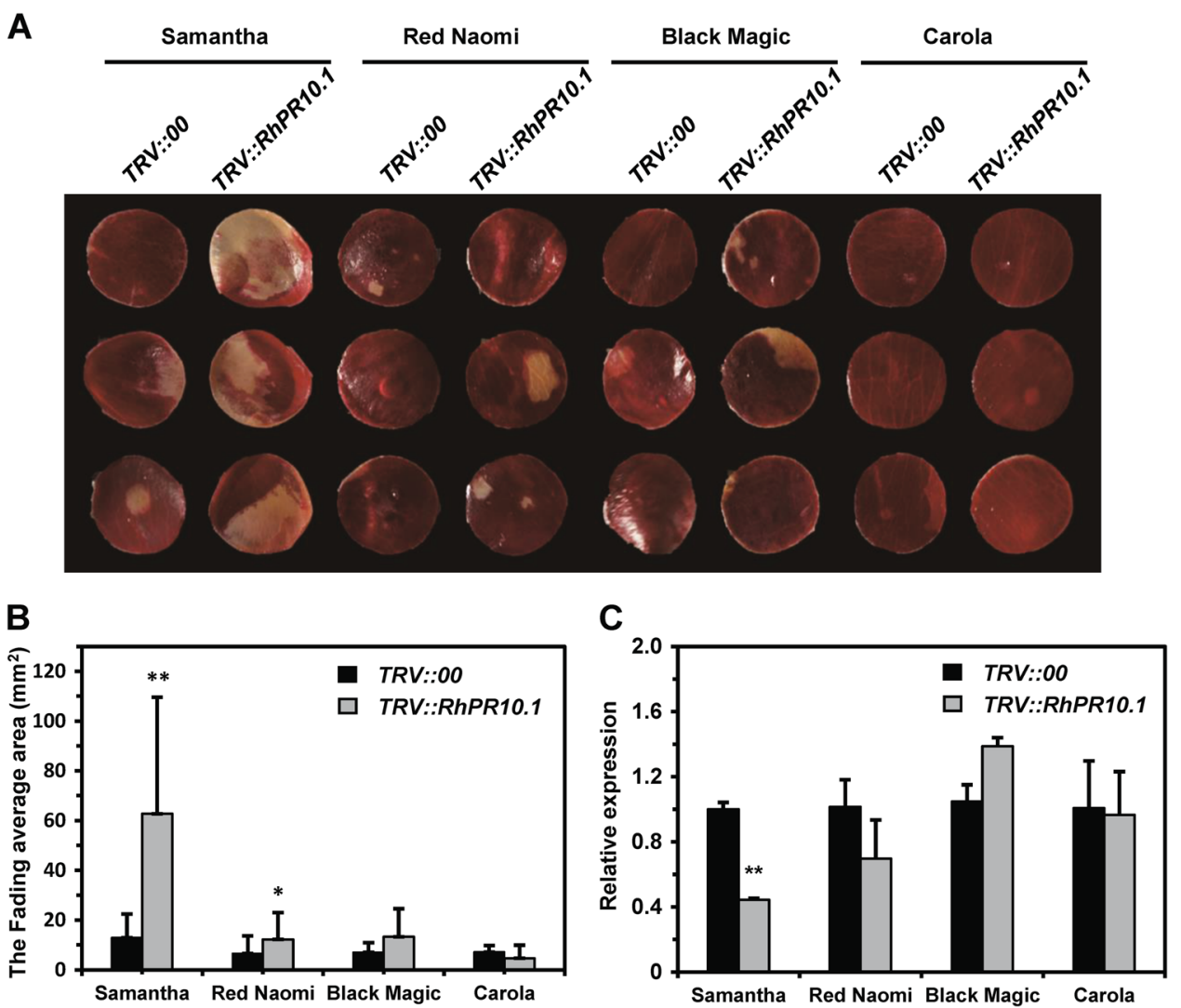

Fig. 3 Comparison of silencing efficiency in the cultivars 'Samantha', 'Red Naomi', 'Black Magic', and 'Carola'. a The strongest color fading upon RhPR10.1 silencing was observed in 'Samantha'; all photographs were taken at 14 days postinfiltration. b Total area of color fading in the four cultivars. The average size of the color-fading area from three biological replicates of each cultivar $(N=48)$ with the standard deviation is shown. c Relative expression of RhPR10.1 at 14 days postsilencing. RhACT5 was used as an internal housekeeping gene control. Statistical analysis was performed using Student's $t$ test; ${ }^{*}, P<0.05 ;{ }^{* *}, P<0.01$.

cultivars to silence their $R h P R 10.1$ genes. At 2 weeks postinfiltration, RhPR10.1-silenced 'Samantha' and 'Red Naomi' petal discs showed significantly increased fading compared with control petals, while the other two cultivars showed no increase in fading (Fig. 3a, b). We further used qPCR to detect the silencing efficiency in the four cultivars. We found that 'Samantha' showed the most significant decrease in RhPR10.1 expression upon infiltration with $T R V:: R h P R 10.1$ (Fig. 3c). These results were consistent with the observation that fading was most pronounced in the petal discs of 'Samantha' and suggested that this was the most suitable cultivar for our purposes, as it was appropriate for the DPDA because of its moderate resistance and for VIGS because of its high silencing efficiency.

To determine whether the agroinfiltration of recombinant TRV vectors affected the responses of rose to B. cinerea, petal discs were vacuum infiltrated with water, infiltration buffer, or Agrobacterium carrying recombinant TRV vectors. The infiltrated petal discs were subsequently inoculated with conidia at $1 \times 10^{4} / \mathrm{mL}, 1 \times 10^{5} / \mathrm{mL}$, or $1 \times 10^{6} / \mathrm{mL}$. However, we did not observe a significant difference in the disease lesions of petal discs infiltrated with water, infiltration buffer, or Agrobacterium carrying recombinant TRV at $60 \mathrm{hpi}$ (Supplemental Fig. S4).

\section{JA plays a role in regulating $B$. cinerea resistance in rose petals}

Previous studies have shown that in the model plant Arabidopsis, the phytohormone jasmonic acid (JA) plays key roles in resistance to necrotrophic pathogens (e.g., $B$. cinerea) and the response to mechanical damage, while salicylic acid (SA) often acts antagonistically to $\mathrm{JA}^{17}$.

In our DPDA system, discs are punched from rose petals. To determine if the mechanical damage caused by this process influences $B$. cinerea-mediated changes in endogenous JA levels, we assessed the changes in endogenous JA and SA levels in petal discs during B. cinerea infection. We used the DPDA to determine endogenous JA and SA levels by high-performance liquid chromatography (HPLC) at $48 \mathrm{hpi}$. We found that petal discs infected with $B$. cinerea exhibited a JA content that was 

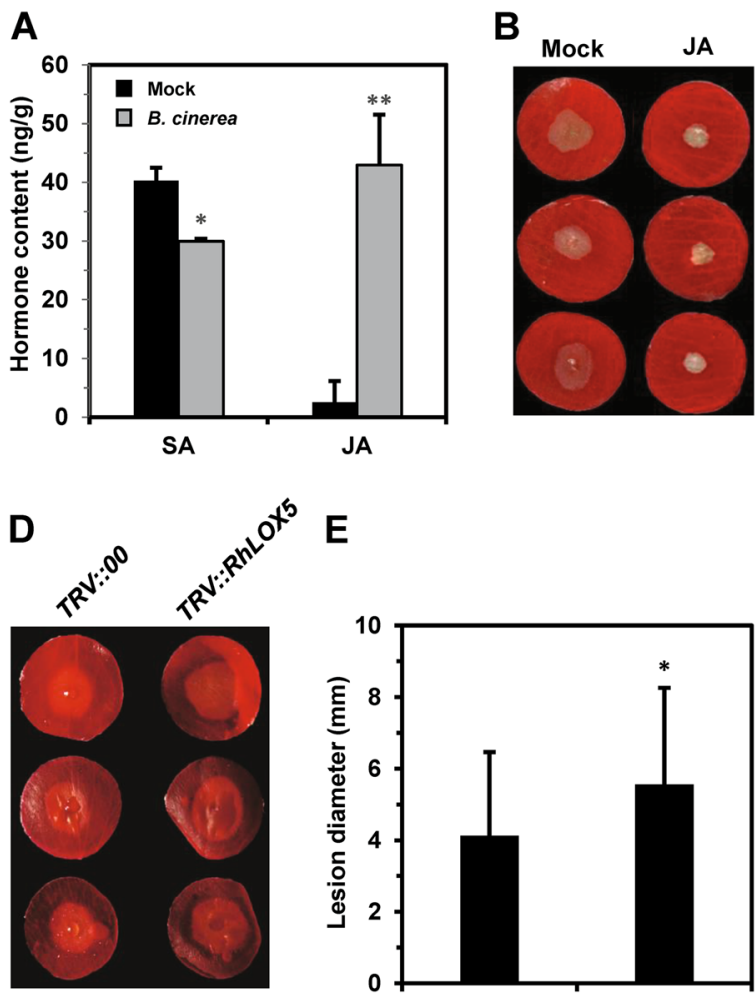

$E$

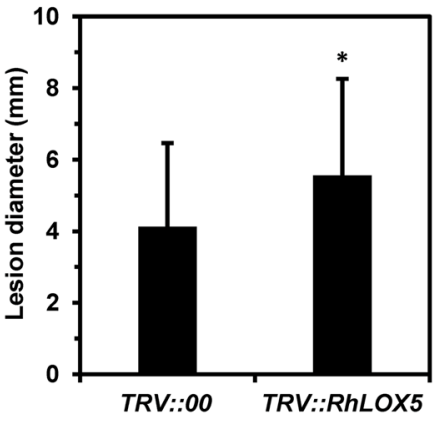

C

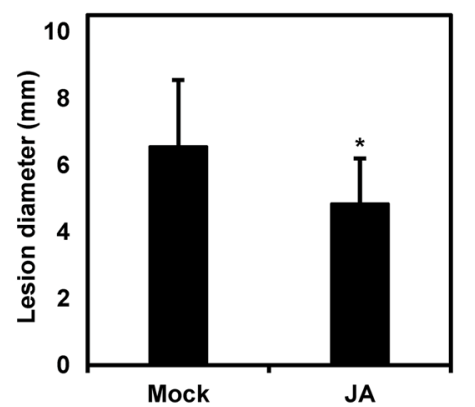

$\mathbf{F}$

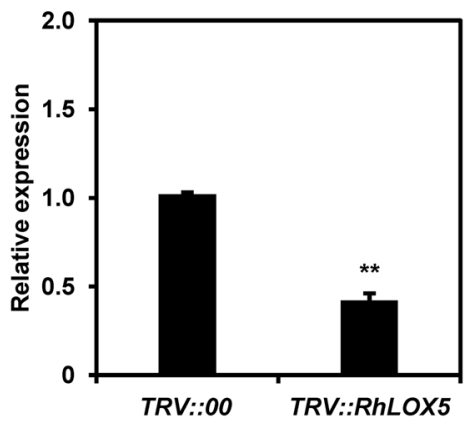

Fig. 4 Jasmonic acid regulates $B$. cinerea resistance in rose petals. a At 48 hpi with B. cinerea, the content of the endogenous hormone JA was significantly increased in petal discs, while that of SA was decreased. $\mathbf{b}$ Exogenous JA treatment significantly enhanced $B$. cinerea resistance in rose petal discs. c Quantification of disease lesions on JA-treated and mock-treated rose petal discs. The graph shows the average diameter of disease lesions at $60 \mathrm{hpi}$; data are from at least three biological replicates of each cultivar $(N \geq 48)$ with the standard deviation. $\mathbf{d}$ Compromised B. cinerea resistance upon silencing of $R h L O X 5$, which encodes a key enzyme of the JA biosynthesis pathway. (e) Quantification of $B$. cinerea disease lesions on $T R V:: R h L O X 5$ - and TRV::00-inoculated rose petal discs. The graph shows the average diameter of the disease lesions at $60 \mathrm{hpi}$; data are from at least three biological replicates of each cultivar $(N \geq 48)$. (f) Relative expression of RhLOX5 at 6 days postsilencing. All statistical analyses were performed using Student's $t$-test; ${ }^{*}, P<0.05 ;{ }^{*}, P<0.01$; hpi hours postinoculation.

almost 10 times higher than that of mock-inoculated discs, whereas the SA content was significantly reduced (Fig. 4a). These results showed that although the DPDA causes mechanical damage to petals, $B$. cinerea infection induces significant changes in phytohormone content in petal discs. The petal discs are therefore representative of the entire plant in these experiments. These results further suggest that JA plays an important role in defense responses in rose petals, as in Arabidopsis.

To test this possibility, we applied $50 \mu \mathrm{M}$ exogenous JA to rose petal discs and inoculated them with $B$. cinerea $24 \mathrm{~h}$ later. The diameter of the disease lesions at $60 \mathrm{hpi}$ was significantly decreased in these petal discs compared with control discs that were not treated with JA (Fig. 4b, c). This experiment confirmed that JA plays a key role in rose defense against $B$. cinerea.

To confirm that VIGS can be used to study $B$. cinerea resistance in rose flowers, we silenced a rose lipoxygenase gene, RhLOX5, that encodes a key enzyme of the JA biosynthesis pathway. At one week post-recombinant TRV infection, we inoculated $R h L O X 5$-silenced and control petal discs with $B$. cinerea. Sixty hours after $B$. cinerea inoculation, we determined the level of disease resistance by evaluating lesion diameter. As expected, the silencing of $R h L O X 5$ resulted in significantly larger lesions compared with the control petal discs, indicative of compromised B. cinerea resistance (Fig. 4d, e). We confirmed the silencing efficiency of VIGS by qPCR (Fig. 4f). These results suggested that JA is required for rose resistance against $B$. cinerea and, importantly, that VIGS in rose petal discs can be used to study genes involved in rose resistance to $B$. cinerea. The DPDA combined with VIGS is a rapid and reliable tool for the functional analysis of rose genes involved in pathogen resistance.

The SA content decreased significantly in the petals at 48 hpi with $B$. cinerea. To examine the role of SA in B. cinerea resistance, we applied $50 \mu \mathrm{M}$ or $100 \mu \mathrm{M}$ exogenous SA to rose petal discs and then inoculated the 
discs with $B$. cinerea $24 \mathrm{~h}$ later. However, we found no significant difference between the SA-treated petal discs and control petal discs that were not treated with SA (Supplemental Fig. S5).

The gaseous hormone ethylene is required for $B$. cinerea resistance in rose petals

The gaseous hormone ethylene (ET) is an important signaling component in plant defense against necrotrophic fungi, such as $B$. cinerea $^{17}$. In Arabidopsis, $B$. cinerea infection results in enhanced ET biosynthesis, and ET is considered to be a positive regulator of $B$. cinerea resistance. However, it has been recently suggested that ET may be involved in both negative and positive effects on the plant's response to $B$. cinerea, depending on the plant species ${ }^{18}$.

To clarify the role of ET in B. cinerea resistance in rose, we applied various concentrations (from 50 to $400 \mu \mathrm{M}$ ) of 1-aminocyclopropane-1-carboxylic acid (ACC), the direct synthetic precursor of ET, to rose petal discs and then inoculated the discs with $B$. cinerea $24 \mathrm{~h}$ later. All four concentrations of ACC used increased rose resistance to B. cinerea (Fig. 5a, b). These results suggested that ET plays a positive role in regulating resistance to $B$. cinerea in rose petals. We further treated petal discs with ET gas or 1-methylcyclopropene (1-MCP, an inhibitor of ET that has been used commercially as a postharvest preservative to maintain cut flower freshness) and inoculated the discs at $24 \mathrm{~h}$ posttreatment. As expected, ET-treated petal discs showed significantly increased resistance, while 1-MCPtreated petal discs showed compromised resistance, confirming the involvement of ET in $B$. cinerea resistance signaling in rose (Fig. $5 \mathrm{c}-\mathrm{f}$ ). Our results suggested that 1-MCP treatment may have a negative effect on rose resistance to $B$. cinerea.

Next, we used VIGS to silence an ET-related gene in rose petals to further demonstrate that the ET signaling pathway plays an important role in the interaction of rose

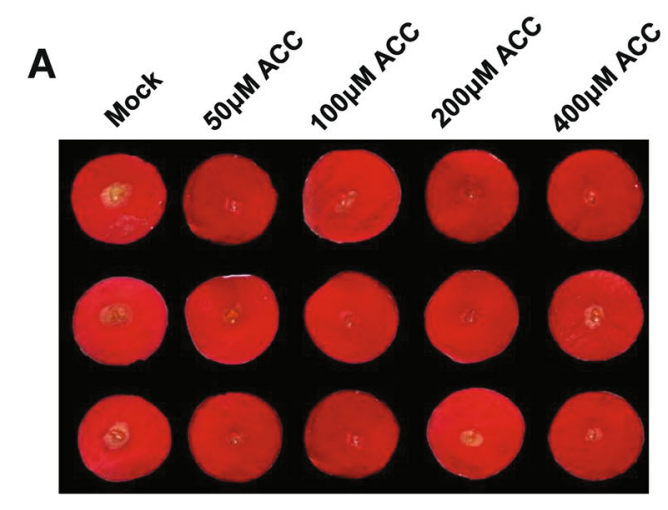

C

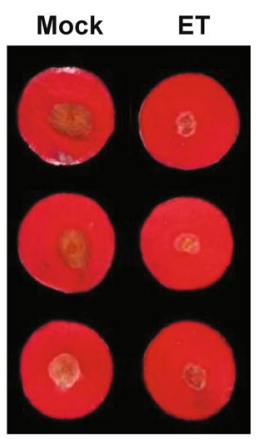

D

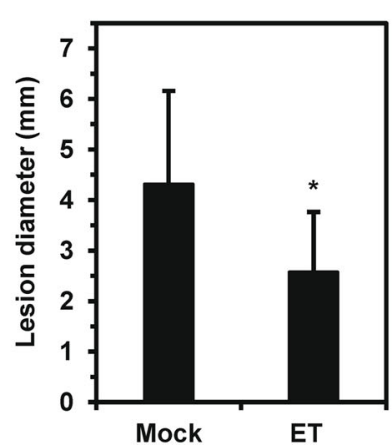

B

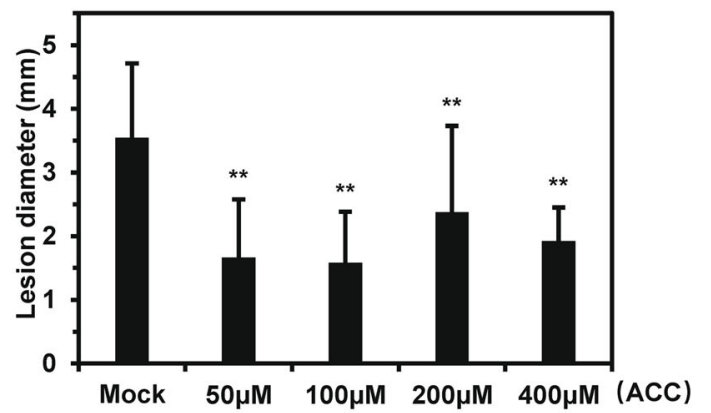

E Mock 1-MCP

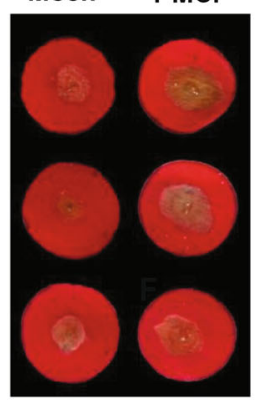

$\mathbf{F}$

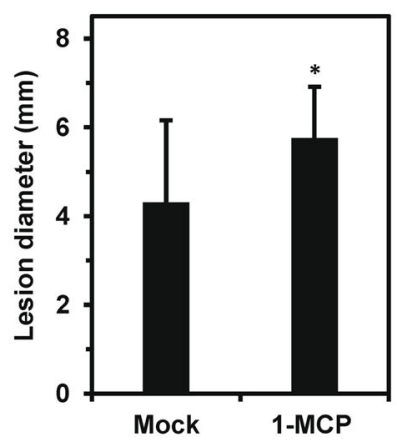

Fig. 5 Ethylene is a key regulator of $\boldsymbol{B}$. cinerea resistance in rose petals. a Exogenous application of ACC, the direct synthetic precursor of ethylene, enhanced B. cinerea resistance in rose petals. $\mathbf{b}$ Quantification of disease lesion size on rose petals treated with ACC or mock-treated rose petals. The graph shows the average diameter of the disease lesions at 60 hpi. c Application of exogenous ethylene (ET) resulted in enhanced $B$. cinerea resistance in rose petals. $\mathbf{d}$ Quantification of disease lesion size in rose petal discs treated with ET or mock-treated rose petals. The average diameter of disease lesions at $60 \mathrm{hpi}$ is shown. e Exogenous application of the ET suppressor 1-MCP compromised B. cinerea resistance in rose petals. f Quantification of disease lesion size on rose petals treated with 1-MCP or mock-treated rose petals. The average diameter of disease lesions at 60 hpi is shown. All data are from at least three biological replicates $(N \geq 48)$ with the standard deviation. All statistical analyses were performed using Student's $t$ test; ${ }^{*}, P<0.05 ;{ }^{* *}, P<0.01$. 


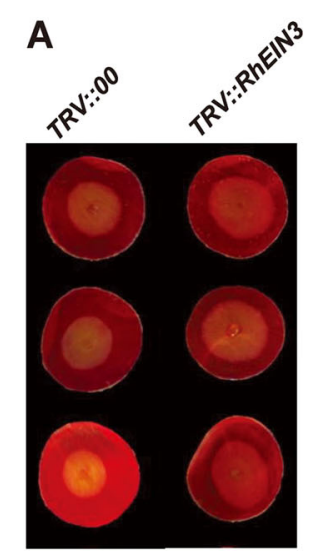

B

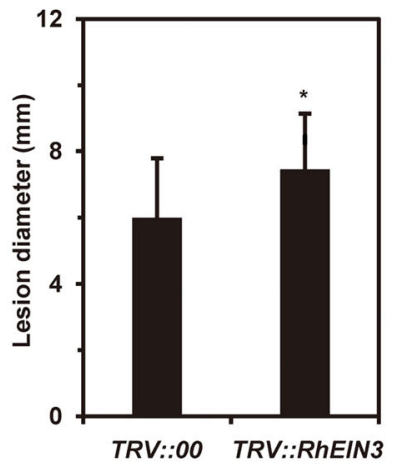

C

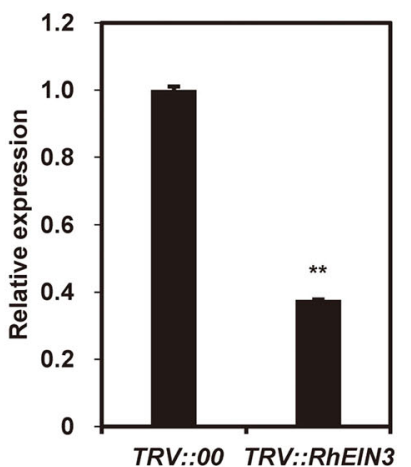

Fig. 6 B. cinerea inoculation of rose petal discs after virus-induced gene silencing of RhEIN3. a Petal discs from the rose cultivar 'Samantha' were inoculated with empty tobacco rattle virus (TRV) as a control (TRV::00) or with a recombinant TRV targeting RhEIN3 (TRV::RhEIN3). Compromised resistance to $B$. cinerea was observed in RhEIN3-silenced plants at 60 hpi. b Compromised $B$. cinerea resistance upon silencing of RhEIN3, which encodes an ethylene regulatory protein. (c) Quantification of RhEIN3 expression in TRV::RhEIN3-inoculated petal discs relative to that in the control. Statistical analysis was performed using Student's t-test; ${ }^{*}, P<0.05 ;{ }^{*}, P<0.01$.

with B. cinerea. In Arabidopsis, the nuclear transcription factor EIN3 is a critical regulator of ET, and the ethyleneinsensitive mutant ein 3 shows increased susceptibility to B. cinerea $^{19}$. To silence EIN3 expression in rose, we inoculated the petal discs with a recombinant virus targeting RhEIN3 expression ( $p T R V:: R h E I N 3)$ or with the empty TRV vector as a control ( $p T R V:: 00)$. As expected, RhEIN3-silenced plants showed significantly compromised resistance (Fig. 6), confirming the positive involvement of $\mathrm{ET}$ in $\mathrm{B}$. cinerea resistance in rose. This further suggested that the DPDA and VIGS can be used for studying ET-related defense genes in rose petals.

\section{Functional analysis of $B$. cinerea-induced transcription factor genes of rose by VIGS and the DPDA}

Previously, 188 B. cinerea-induced transcription factor $(B I T)$ genes were identified via comparative RNA-seq analysis of rose flowers infected with $B$. cinere $^{2}$. To illustrate the potential application of our methods to a high-throughput reverse screen of gene function in rose, we performed VIGS to knock down the expression of 10 randomly selected $B I T$ genes. To this end, we cloned fragments of selected $B I T$ genes varying in length from 200 to $400 \mathrm{bp}$ into the TRV vector and inoculated rose petal discs with these fragments via vacuum infiltration. To test the involvement of the selected BIT genes in $B$. cinerea resistance, we subsequently challenged the $T R V::$ $B I T$-inoculated rose petal discs with $B$. cinerea. The $T R V::$ RhMYB44- and TRV::RhTGA2-inoculated plants showed severely compromised resistance, as evidenced by significantly increased lesion size (Fig. 7). These results indicated that $R h M Y B 44$ and $R h T G A 2$ are essential for $B$. cinerea resistance in rose. Furthermore, VIGS in combination with the DPDA provided a rapid and highthroughput method for assessing the functions of rose genes in disease resistance in petals.

\section{Discussion}

The DPDA is a versatile tool for comparative analyses of $B$. cinerea resistance in rose

In the present study, we developed a DPDA that enabled us to quantitatively evaluate $B$. cinerea resistance in rose flowers. Importantly, the results of the DPDA in resistant and susceptible cultivars matched their resistance abilities observed in the field, suggesting that the DPDA is a reliable method for quantifying resistance against $B$. cinerea in rose flowers.

Compared with whole-flower inoculation, our DPDA has clear advantages. First, petal discs are easy to inoculate, as they are relatively flat, whereas rose petals on intact flowers are cambered, so that inoculum drops tend to roll down the flower rather than remain on the petal. Second, the petal discs used in the DPDA require much less space in the climate chamber than do cut flowers in vases. Finally, that detached petal discs could be left on agar plates for over 10 days without dehydration, while the vase life of cut roses is significantly shorter, with cut flowers wilting within a week. This is important because, although $B$. cinerea lesions develop within 3 days, pretreatment with phytohormones or VIGS takes additional time.

Originally, we also considered the use of detached intact petals for the inoculation assay; however, similar to intact flowers, the petals are cambered and, thus, more difficult to inoculate. In addition, intact petals wilt more easily than discs on $0.4 \%$ agar plates. Finally, using the entire 


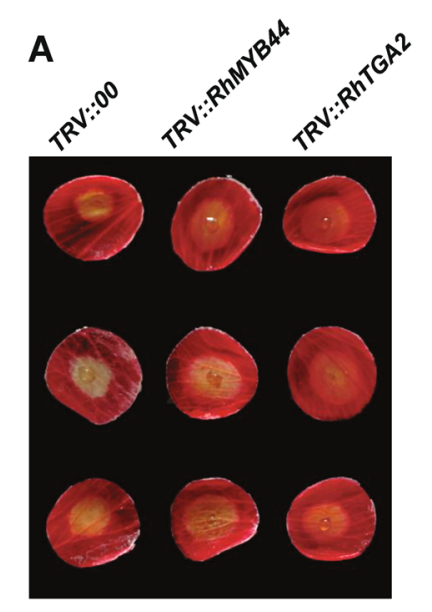

B

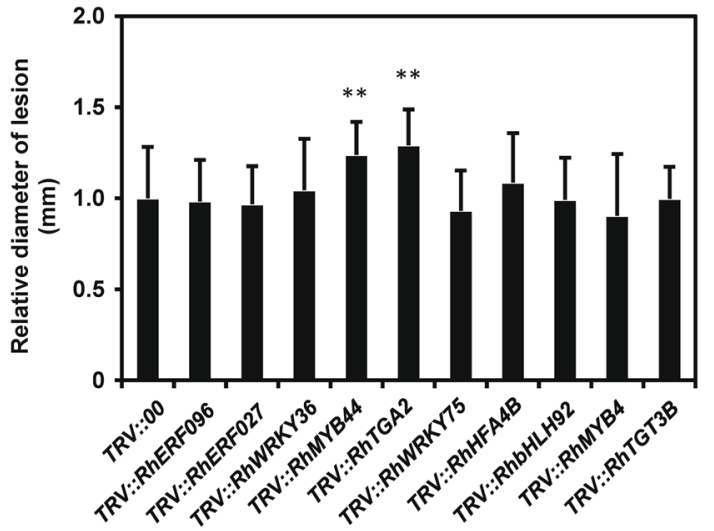

Fig. 7 Functional screen of $B$. cinerea-induced rose transcription factor genes by VIGS and the DPDA. a Compromised $B$. cinerea resistance symptoms on rose petal discs upon infection with a recombinant tobacco rattle virus (TRV) targeting RhMYB44 and RhTGA2, shown at 60 hpi. b Quantification of disease lesions on BIT-silenced plants. The graph shows the average diameter of disease lesions with the standard deviation at 60 hpi relative to the control (TRV::00). Statistical analysis was performed using Student's $t$ test; ${ }^{*}, P<0.05 ;{ }^{*}, P<0.01$.

petal takes up more space in the climate chamber, as only one petal can be placed in a 9-cm petri dish, whereas 16 discs (i.e., 16 independent inoculation repeats) can fit in a petri dish of this size.

A potential disadvantage of the DPDA is that the mechanical damage caused by punching induces the accumulation of JA, which is also a resistance signal against necrotrophic fungal pathogens, such as $B$. cinerea. However, we circumvented this concern by including proper controls. For example, we found that petal discs inoculated with $B$. cinerea accumulated significantly higher levels of JA (almost 10 times as high; Fig. 4a) than mock-inoculated discs prepared in the same fashion, indicating that detached petal discs are still very sensitive to $B$. cinerea and that the defense signaling molecule JA is further induced upon infection beyond the levels caused by the process of obtaining discs.

\section{TRV-based VIGS for the rapid and high-throughput screening of rose genes in $B$. cinerea resistance}

VIGS is a rapid and powerful tool for reverse genetics in various plant species, especially those in which genetic transformation is inconvenient or laborious. In rose, we previously devised a grafting VIGS method in which axillary sprouts were cut off and vacuum infiltrated. The inoculated scions were then grafted back onto the plants for flowering. Flower silencing phenotypes could be observed within four or five weeks of infiltration. We have shown that this grafting VIGS method can be used to efficiently silence genes related to floral color, development, and fragrance, providing a relatively fast method for assessing the functions of genes in roses $^{12}$.
Grafting VIGS in intact rose flowers can certainly also be used to study $B$. cinerea resistance. However, the establishment of VIGS in intact rose flowers takes several weeks (until the agroinoculated sprout flower) and requires large-scale facilities for growing many plants and a great deal of work. By contrast, the silencing of candidate genes in petal discs can be accomplished in a matter of days and uses fewer resources. In the present study, by silencing RhLOX5 and EIN3, which are genes required for JA- and ET-mediated resistance to $B$. cinerea, respectively, we demonstrated that the DPDA in combination with VIGS in petal discs can be used to elucidate defense signaling against $B$. cinerea in rose flowers. In addition, although the silencing of RhDFR1 induces bleached petals in complete rose flowers ${ }^{12}$, this gene is not a suitable VIGS reporter for petal discs. However, we showed that the transient silencing of RhPR10.1 promoted accelerated color-fading phenotypes of petal discs, suggesting that this gene can be used as a visible reporter for VIGS in petal discs.

TRV-derived VIGS has been successfully applied to many plant species. However, the high-throughput screening of gene function using VIGS remains challenging in many plant species owing to time, labor, and space requirements. A high-throughput screen of gene function by VIGS has been reported only in $N$. benthamiana, in which it was originally developed ${ }^{20,21}$. Considering that agroinoculated petal discs can be conveniently and efficiently silenced within several days and require relatively little space in a climate room, the VIGS and DPDA methods described herein can potentially be used not only for the functional analysis of a single gene but also for high-throughput screening of a large number of candidate 
genes involved in B. cinerea resistance. Indeed, in this study, we used the DPDA combined with VIGS to identify two novel transcription factor genes not previously known to be involved in $B$. cinerea resistance in rose.

\section{Materials and methods Plant materials}

Rosa hybrida 'Samantha' plants were grown in soil in the greenhouse under the following conditions: 20 to $25{ }^{\circ} \mathrm{C}$ with $50 \%$ to $70 \%$ relative humidity and $16 \mathrm{~h} / 8 \mathrm{~h}$ day/night periods. Cut roses were harvested from the greenhouse at stage 2 of flower opening and immediately placed in water. All other rose cultivars were ordered from the Kunming International Flora Auction Trading Center and delivered to the lab within $24 \mathrm{~h}$. The flower stems were recut to $20 \mathrm{~cm}$ in length under water and placed in deionized water. After treatment, the flowers were placed in a vase with deionized water under controlled conditions of $23-25^{\circ} \mathrm{C}$ with $30-40 \%$ relative humidity and $12 \mathrm{~h} / 12 \mathrm{~h}$ day/night periods to await further processing.

\section{B. cinerea inoculation}

B. cinerea strain B05.10 or CAU8324 was grown on potato dextrose agar at $22^{\circ} \mathrm{C}$. B. cinerea conidia were harvested from 10- to 14-day-old fungal plates and washed with tap water. The conidia were suspended at the desired final concentration in half-strength potato dextrose broth (PDB). For inoculation, rose petals were punched into $16 \mathrm{~mm}$ discs and rinsed with deionized water. Then, $2 \mu \mathrm{L}$ of a conidial suspension was dropped onto the adaxial surface of the punched petals. As a control, petals were mock-inoculated with half-strength PDB. After (mock) inoculation, the petals were transferred to agar plates. The inoculated petals were evaluated by observing disease symptoms at $60 \mathrm{hpi}$. Each inoculation was repeated at least three times using a total of at least 48 discs. Student's $t$ test was used for statistical analysis. For the measurement of ion leakage, petal discs from each treatment were placed in $10 \mathrm{~mL}$ of $0.4 \mathrm{~m}$ mannitol and shaken at $40 \mathrm{rpm}$ for $3 \mathrm{~h}$. A conductivity meter (DDBJ-350; LeiCi) was used to measure the conductivity of the solution.

\section{Detection of phytohormones in rose petals}

The plant samples were ground to a powder in liquid nitrogen. Then, $500 \mu \mathrm{L}$ of extraction buffer (2-propanol/ $\mathrm{H}_{2} \mathrm{O} / \mathrm{HCl}$ [2:1:0.002, v/v/v]) was added, and the samples were vortexed for $10 \mathrm{~s}$ and shaken for $30 \mathrm{~min}$ at $4{ }^{\circ} \mathrm{C}$. Next, $1 \mathrm{~mL}$ of $\mathrm{CHCl}_{3}$ was added, and the solution was vortexed for $10 \mathrm{~s}$, shaken for $30 \mathrm{~min}$ at $4{ }^{\circ} \mathrm{C}$, and then centrifuged at $14,000 \mathrm{rpm}$ for $5 \mathrm{~min}$ at $4{ }^{\circ} \mathrm{C}$. Finally, $1.2 \mathrm{~mL}$ of the lower layer of the liquid was transferred to a fresh test tube and dried at room temperature under nitrogen.
The dried sample was resuspended in $0.1 \mathrm{~mL}$ of methanol and assayed by liquid chromatography-mass spectrometry (HPLC Shim-pack UFLC SHIMADZU CBM30A system; MS, Applied Biosystems 6500 Triple Quadrupole).

\section{Hormone treatments of rose flowers}

For the hormone treatments, flower stems were placed in vases containing aqueous solutions of JA $(50 \mu \mathrm{M})$, salicylic acid (50 and $100 \mu \mathrm{M})$, or 1-aminocyclopropane1-carboxylic acid (ACC; 50, 100, 20, and $400 \mu \mathrm{M}$ ), or in deionized water as a control, for $24 \mathrm{~h}$. The flower petals were then punched into discs for inoculation.

\section{Ethylene and 1-MCP treatment of rose flowers}

Ethylene and 1-MCP were applied to the roses as described previously ${ }^{13}$. Briefly, rose flowers were exposed to ethylene $(10 \mu \mathrm{L} / \mathrm{L}), 1-\mathrm{MCP}(2 \mu \mathrm{L} / \mathrm{L})$, or regular air as a control for $24 \mathrm{~h}$. A $1 \mathrm{M} \mathrm{NaOH}$ solution was added to the chambers to prevent $\mathrm{CO}_{2}$ accumulation. The flower petals were then punched into discs for inoculation.

\section{VIGS}

To obtain the TRV::RhLOX5 construct, a 461-bp fragment from the 3' UTR of RhLOX5 was cloned into the TRV vector $p T R V 2$ and then electroporated into Agrobacterium strain GV3011. The TRV constructs TRV::00, $T R V:: R h P R 10.1$, and TRV::RhEIN3 have been described previously ${ }^{15,16,22}$. To establish VIGS in rose petals, detached petals were obtained from the outermost whorls of rose flowers at stage 2 of flower opening. Then, a 15$\mathrm{mm}$ disc was punched from the center of each petal. The petal discs were vacuum infiltrated with Agrobacterium carrying TRV constructs as described by Zhang and Thomma ${ }^{14}$. Briefly, an overnight culture of Agrobacterium was harvested at an $\mathrm{OD}_{600}$ of 0.8 by centrifugation at $4000 \mathrm{rpm}$ for $1 \mathrm{~min}$ and then resuspended in the infiltration buffer (IB; $20 \mathrm{~g}$ sucrose, $5 \mathrm{~g}$ Murashige and Skoog medium without vitamins, $10 \mathrm{~mL}$ of $1 \mathrm{M} \mathrm{N}$-morpholinoethanesulfonic acid, $1 \mathrm{~mL}$ of $0.2 \mathrm{M}$ acetosyringone) to a final $\mathrm{OD}_{600}$ of 2. Agrobacterium cultures containing constructs expressing $T R V 1$ and $T R V 2$ were mixed in a 1:1 ratio and vacuum infiltrated into petal discs. The petal discs were immersed in the bacterial suspension, and infiltration was performed under vacuum at $0.7 \mathrm{MPa}$. After the release of the vacuum, infiltrated petal discs were washed with deionized water. After TRV infection, the petal discs were left under dark conditions at $8^{\circ} \mathrm{C}$ for 3 days. Then, the discs were placed in a climate chamber at $22{ }^{\circ} \mathrm{C} / 19^{\circ} \mathrm{C}$ with $16 \mathrm{~h} / 8 \mathrm{~h}$ day/night periods for 3 days. At 6 days post-TRV infection, the petal discs were inoculated with $B$. cinerea. VIGS was repeated at least three times using at least 16 discs in each experiment. After $B$. cinerea inoculation, Student's $t$-test was conducted. All primers are listed in Supplemental Table S1. 


\section{RNA extraction and qPCR}

Total RNA was extracted from rose petal discs as described previously ${ }^{13}$. First-strand cDNA was synthesized from $1 \mu \mathrm{g}$ of total RNA using the Takara Reverse Transcriptase M-MLV cDNA Synthesis Kit (Takara, Dalian, China) according to the manufacturer's instructions. One microliter of first-strand cDNA was used as a template in the reaction with the KAPA SYBR Quantitative PCR Kit (Kapa Biosystems), which was run in a StepOnePlus Real-Time PCR System (Thermo Fisher Scientific). RhACT5 was used as an internal housekeeping gene control as described previously ${ }^{12}$. All primers used for qPCR are listed in Supplemental Table S1.

\section{Acknowledgements}

Z.Z. is supported by the National Natural Science Foundation of China (31772344 and 31501791). This research was funded in part by a grant from the Natural Science Foundation of Beijing Municipality (6162017) to Z.Z.

\section{Authors' contributions}

X.C., H.Y., X.L., and Z.Z. contributed to the experimental design. X.C., H.Y., X.L, D.L., M.S., J.W., and H.Y. carried out the experiments, M.J.M.S., Y.B., and Z.Z. interpreted the results. Z.Z. prepared the manuscript. M.J.M.S., Y.B., and Z.Z. edited the manuscript.

\section{Conflict of interest}

The authors declare that they have no conflict of interest.

Supplementary Information accompanies this paper at (https://doi.org/ 10.1038/s41438-019-0219-2).

Received: 18 June 2019 Revised: 30 September 2019 Accepted: 23 October 2019

Published online: 01 December 2019

\section{References}

1. Qi, W. et al. Genomic and transcriptomic sequencing of Rosa hybrida provides microsatellite markers for breeding, flower trait improvement and taxonomy studies. BMC Plant Biol. 18, 119 (2018).

2. Liu, X. et al. Comparative RNA-Seq analysis reveals a critical role for brassinosteroids in rose (Rosa hybrida) petal defense against Botrytis cinerea infection. BMC Genet. 19, 62 (2018).

3. Williamson, B., Tudzynski, B., Tudzynski, P. \& van Kan, J. A. Botrytis cinerea: the cause of grey mould disease. Mol. Plant Pathol. 8, 561-580 (2007).
4. Hao, Y. et al. Potential applications and antifungal activities of engineered nanomaterials against gray mold disease agent Botrytis cinerea on rose petals. Front. Plant Sci. 8, 1332 (2017).

5. Williamson, B. et al. Effect of humidity on infection of rose petals by dryinoculated conidia of Botrytis-Cinerea. Mycol. Res. 99, 1303-1310 (1995).

6. Fu, Y. et al. Genetic mapping and QTL analysis of Botrytis resistance in Gerbera hybrida. Mol. Breed. 37, 13 (2017).

7. Elad, Y. Latent infection of Botrytis-cinerea in rose flowers and combined chemical and physiological control of the disease. Crop Prot. 7, 361-366 (1988).

8. Lu, P. et al. RhHB1 mediates the antagonism of gibberellins to ABA and ethylene during rose (Rosa hybrida) petal senescence. Plant J. 78, 578-590 (2014).

9. Pie, K. \& Brouwer, Y. J. C. M. Susceptibility of cut rose flower cultivars to infections by different isolates of Botrytis cinerea. J. Phytopathol. 137, 233-244 (1993).

10. Morandi, M. A. B., Sutton, J. C. \& Maffia, L. A. Effects of host and microbia factors on development of Clonostachys rosea and control of Botytis cinerea in Rose. Eur. J. Plant Pathol. 106, 439-448 (2000).

11. Liu, Y. L., Schiff, M. \& Dinesh-Kumar, S. P. Virus-induced gene silencing in tomato. Plant J. 31, 777-786 (2002).

12. Yan, H. et al. Graft-accelerated virus-induced gene silencing facilitates functional genomics in rose flowers. J. Integr. Plant Biol. 60, 34-44 (2018).

13. $\mathrm{Ma}, \mathrm{N}$. et al. Rh-PIP2;1, a rose aquaporin gene, is involved in ethylene-regulated petal expansion. Plant Physiol. 148, 894-907 (2008).

14. Zhang, Z. \& Thomma, B. Virus-induced gene silencing and tumefaciensmediated transient expression in Nicotiana tabacum. Methods Mol. Biol. 1127, 173-181 (2014).

15. Zhang, Z. et al. Optimized agroinfiltration and virus-induced gene silencing to study Ve1-mediated Verticillium resistance in tobacco. Mol. Plant Microbe Interact. 26, 182-190 (2013).

16. $\mathrm{Wu}, \mathrm{L}$. et al. An ethylene-induced regulatory module delays flower senescence by regulating cytokinin content. Plant Physiol. 173, 853-862 (2017).

17. Thomma, B. P., Penninckx, I. A., Broekaert, W. F. \& Cammue, B. P. The complexity of disease signaling in Arabidopsis. Curr. Opin. Immunol. 13, 63-68 (2001).

18. AbuQamar, S., Moustafa, K. \& Tran, L. P. Mechanisms and strategies of plant defense against Botnytis cinerea. Crit. Rev. Biotechnol. 37, 262-274 (2017).

19. Song, S. et al. Interaction between MYC2 and ETHYLENE INSENSITIVE3 modulates antagonism between jasmonate and ethylene signaling in Arabidopsis. Plant Cell. 26, 263-279 (2014).

20. Lu, R. et al. High throughput virus-induced gene silencing implicates heat shock protein 90 in plant disease resistance. EMBO J. 22, 5690-5699 (2003).

21. Wang, Y. et al. Leucine-rich repeat receptor-like gene screen reveals that Nicotiana RXEG1 regulates glycoside hydrolase 12 MAMP detection. Nat. Commun. 9, 594 (2018).

22. Luo, J. et al. A DELLA gene, RhGAl1, is a direct target of EIN3 and mediates ethylene-regulated rose petal cell expansion via repressing the expression of RhCesA2. J. Exp. Bot. 64, 5075-5084 (2013). 